\title{
Naval ship recognition
}

\author{
I. Camino García and U. Zölzer \\ Helmut-Schmidt-Universität/University of the Federal Armed Forces, Department of Signal Processing \\ and Communications, Hamburg, Germany \\ Correspondence to: I. Camino García (irene.camino@hsu-hh.de)
}

\begin{abstract}
Object recognition is a very interesting task with multiple applications and for that reason it has been dealt with very intensively in the last years. In particular, the application to naval ship pictures may facilitate the work of the coastguards or the navy. However, this type of images entails some difficulties due to their specific environment. Water reflects the light and as a consequence, some areas may presumably show different brightness and color. Waves from wind or moving ships pose a problem due to the additional edges that they produce. The camouflage of ships in the military context is also an issue to take into account. Therefore, it is difficult to propose a simple method that is valid for every image.

A discussion about which techniques may solve these problems is presented and finally a combined solution based on contour recognition is suggested. Test images are preprocessed by histogram stretching. Then, the Canny method is applied to the image and to the reference contour in order to obtain not only their edges, but also their respective orientations. The problem of recognizing the reference contour within the detected edges is addressed by making use of the Generalized Hough Transform (GHT).
\end{abstract}

\section{Introduction}

The problem shown in this study is a particular case of how to find an object in an image, so that it involves image processing. By applying these methods, certain image properties are manipulated to obtain an improved version of the input as output. This improvement must be understood as the required enhancement by a specific application. It will not necessarily fulfill the requirements of another purpose.

Image processing is employed in multiple fields in order to reach different goals. Some examples are criminology, meteorology, biomedicine or industrial automation. Sometimes it is enough to obtain a better representation of the image re- lated to its level of brightness or to the colours. However, a more complex process is carried out for object recognition.

Nowadays the automation of processes is continually being requested and not only by the industry, but also by the users. It can be addressed by object recognition because it allows to compile information without human intervention. It also helps identify camouflaged objects that are difficult to be distinguished by the human eye. Therefore it could facilitate the process of registration of ships coming or going out of a port, the work of coastguards or the navy, or for ship classification. In case that the interest was the search of a specific ship, the operator in charge could base the decision on a processed image, more visual than the original one. However, this work intends to be part of a further system of classification. Having a database that contains the models of interest, the following technique would be applied to each of them. After a decision stage, the ship model of the input image would be automatically identified.

The different issues of naval images are studied in Sect. 2 as well as the possible techniques to employ. In Sect. 3, the Generalized Hough Transform (GHT) is also explained to better understand later, in Sect. 4, which aspects could be improved. A simple preprocessing technique is proposed in Sect. 5. Finally, the results and the conclusion are discussed in Sects. 6 and 7, respectively.

\section{Analysis of the problem}

Since the final task is the recognition of a ship within an image (see Fig. 1), it is essential to consider what this involves.

Very often, the ship is composed of several regions with different grey levels, caused by the incidence of light. This hinders from recognizing all of them together as a single object. False detections or missed detections are likely to occur, so region based segmentation can not be suggested here. 
However, in addition to these regular object recognition problems, naval ship images entail specific issues due to their nature.

It is important to bear in mind that naval ships are often camouflaged. Then, the difference of intensity between them and the background might be small. Other pictures may have textured background due to reflections of the water. And sometimes, even the ship will be reflected on the surface. These reasons show that it is very difficult to propose a fixed threshold or interval for histogram based methods.

As result of this special environment, waves will make the waterline of the same ship vary. They will also produce a large amount of edges and edge linking techniques may get lost. In order to preserve the semantic content of the ship, edge detectors will not be valid or, at least, not as final solution.

For these reasons the ship will be represented by its contour and it will be fitted along the input image with the help of the Generalized Hough Transform. The Canny edge detector (Canny, 1986) will be responsable of obtaining the edges and their respective orientations, as often suggested in literature (see Duprat et al., 2005 and Tang et al., 2011).

\section{Generalized Hough Transform (GHT)}

This algorithm is the generalization of the basic Hough Transform (Hough, 1962). The main idea is based on the representation of any shape, and it was developed by Ballard (1981). It has been deeply analyzed in terms of storage (Lee et al., 1992) or robustness and improved by new contributions (see Kassim et al., 1999).

Any kind of shape can be represented as a circle but instead of the center, the shape has a reference point. Once it is fixed, the shape can be seen as a circle but with different values of radium depending on the angle, instead of a constant value. This reasoning allows to apply the Hough Transform for circles to general shapes, as it follows.

A first step is carried out in order to obtain information about the contour where to work on later. A reference point $\boldsymbol{y}=\left(x_{r}, y_{r}\right)$ is chosen to practise the function the circle center. Besides, it will determine the location of the shape in the input image and all the measures will be made with respect to it.

Then, for each boundary point $\boldsymbol{x}$ of the shape $\boldsymbol{r}=\boldsymbol{y}-\boldsymbol{x}$ is calculated. Once the contour of the shape is defined this way, it is indispensable to make use of the orientation, that is provided by an edge detector. As this algorithm is suitable for any shape, the range of orientation values $\phi$ is assumed to be wide. So they are divided into intervals that depend on the required level of precision. Each $\boldsymbol{r}$ is classified in these intervals, according to the gradient angle of its respective $\boldsymbol{x}$.

In summary, there will be a look-up table indexed by these fixed values and in every entry different $\boldsymbol{r}$ will be stored. These $\boldsymbol{r}$ correspond to the points $\boldsymbol{x}$ whose gradient angle is the closest to the index. This table is a very important element because it gathers all the necessary information. This information is also organized, creating the representation of the shape that the algorithm will search later in the input image. The table is called the R-Table and the resolution $n$ can be selected according to the needs of every case.

Then, the match of the contour is perfomed. For every pixel that turned out to be edge point, its gradient angle $\phi$ is calculated. This way it is possible to determine which table entry would correspond them if they were boundary points of the shape. For a particular edge point $\boldsymbol{x}$ of the input image, Eq. (1) is applied to every $\boldsymbol{r}$ that was stored in the corresponding entry. The results are the possible locations of the reference point $\boldsymbol{y}$.

$y=x+r$

It is clear, following the previous reasoning, that the lower the resolution, the more times that this equation will be computed. Therefore the process takes longer because there will be more $\boldsymbol{r}$ in each entry.

An accumulative array $\mathbf{A}$ is in charge of keeping the count of the times that each possible location is the result of the above equation. Every cell represents the value of $\boldsymbol{y}$, so they are accordingly incremented. The cell $\boldsymbol{y}$ that contains the maximum of $\mathbf{A}$ is assumed to represent the position of the reference point.

However the output of the GHT can not represent the reference point of the shape if it does not exist in the input image. In this case, the result will be the reference point of the most similar shape.

There is another possibility in order to make the search of the contour independent of its size or its position. This way if, for example, the shape is rotated a certain angle $\theta$ it is still possible to find it. A range of values of $\theta$ and the factor that will modify the size, $s$, is then established. Obviously the array $\mathbf{A}$ will also depend on this parameters, $s$ and $\theta$.

\section{Applying the GHT}

However, when applying the GHT to this type of images it is important to consider the facts explained in Sect. 2.

The first decision made was the location of the reference point $\boldsymbol{y}$. In this case, inside the shape and at the half of the width and height of the ship model to reduce rounding errors.

Then the Canny edge detector supplies the edge points of both, input image and shape. The thresholds for the hysteresis are set to 64 and 128 .

The large amount of unwanted edge points, especially due to waves, will cause an excessive amount of calculations. As long as the orientations of the edge points belong to any interval, they will compute the equations and perform the voting process. This will not only delay the process but unnecesary contributions in $\mathbf{A}$ will also take place. 
Furthermore, the shape of any ship is complex, with a large variety of orientation values. Hence it is unlikely to find empty entries in the R-Table, what would directly reject edge points. The number of $\boldsymbol{r}$ that fill each row is also too high.

Then, the ideal case would be to devote an entry to each value of orientation present in the ship model. The resolution $n$ would be the number of boundary points in the ship model if they all have different orientations. By limiting the possible values of the index, infinite values of $\phi$ would be rejected when building the R-Table. This way the number of elements of each entry would decrease. Only edge points with exactly the same orientation that the boundary points would compute the Eq. (1).

However, the Canny operator makes use of the surrounding pixels to calculate the orientation of a certain pixel. As mentioned before, the background is not constant in its grey value but it changes tremendously, on the contrary to other applications as license plates or X-rays images. So the edge points in the input image will not eventually have the same orientation as the corresponding boundary points of the shape, but slightly different. This difference will cause the equation not to be evaluated for these edge points and the corresponding voting process will not be carried out.

A fixed range $a$ may be assigned to every orientation of the boundary points $\phi_{i} \pm a, i=0, \ldots, n-1$. Assuming that the orientations of the corresponding edge points will belong to it, this contributes to some flexibility instead. A large amount of unwanted edge points would be still rejected too. The number of missed detections depend on the grey distribution of the background of each image, and therefore this range $a$. So, despite this extension, missed detections still occur for some images unless the value of $a$ is high enough. Setting $a$ to cover all cases might cause the overlap, i.e. $\phi_{i}+a>$ $\phi_{i+1}-a$, between some or even all intervals though.

In order to avoid overlaps the range of orientations needs to be completely covered. The resolution will be set up looking for a balance between a high value to better spread the different $\boldsymbol{r}$ and a small value to avoid missed detections. In the end, the selected resolution was $n=8$.

This analysis shows the need of reducing the number of unwanted edges and the dispersion between the orientations of the boundary points and the corresponding edge points of the input image. For that purpose, a preprocessing step is suggested in the following section.

\section{Preprocessing}

The preprocessing step proposed works well for this kind of images, because of the assumptions made to apply it.

The image is divided into three horizontal areas, where the top and the bottom ones are made up of the fourth part of the total number of pixels of the image. Then, the ship is supposed to be located in the central part. As the ship and
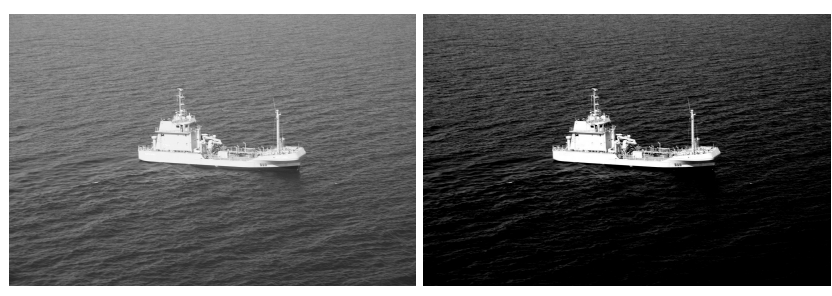

(a)
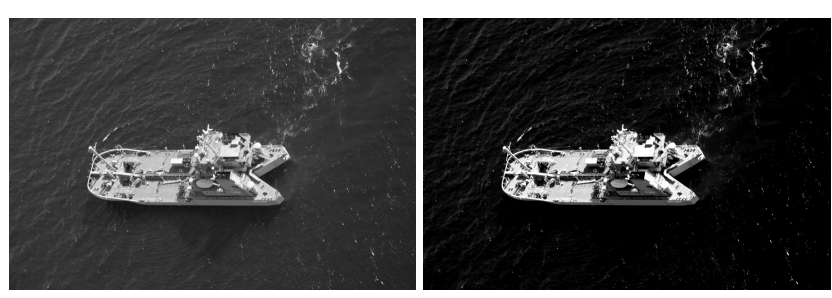

(b)
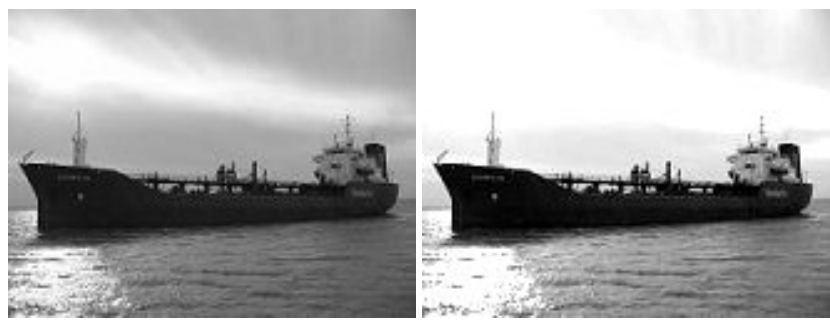

(c)

Fig. 1. Input images before (left) and after (right) the preprocessing step.

the background are intended to have opposite grey values, the histogram stretching is performed with quantiles based on the histogram of the central part. This way the pixel intensities are redistributed as shown in Fig. 1.

Depending on the image, good visual results can be achieved, what might lead to consider that this preprocessing is intended to be a segmentation technique. However, the goal to pursue is the improvement of the issues previously mentioned in a simple, fast way.

\section{Results}

When the GHT is applied to the test images, the output is always the original reference point that was selected in their respective shapes. This is according to what it is expected and it shows that the thresholds of the Canny operator are adequate.

The goal is to improve, for this type of images, other important aspects by solving the problems that were previously 
Table 1. Reduction in edge points after the preprocessing step (\%).

\begin{tabular}{lr}
\hline Figure 1a & 27.65 \\
\hline Figure 1b & 6.68 \\
\hline Figure 1c & 51.16 \\
\hline
\end{tabular}

Table 2. Reduction in boundary points that were detected when increasing $n=8$ to $n=36(\%)$.

\begin{tabular}{ll}
\hline Figure 1a & 54.02 \\
\hline Figure 1b & 36.34 \\
\hline Figure 1c & 16.28
\end{tabular}

pointed out and discussed. Therefore, the number of edge pixels of each input image and the value of the maximum in A, according to the resolution and the preprocessing step.

An important reduction in the number of edge points a when the preprocessing step is applied, as Table 1 shows. The result of incrementing the resolution, from the selected value $n=8$ to $n=36$, certainly leads to a reduction in the number of points detected as boundary points of the model, as can be noticed in Table 2 .

\section{Conclusions}

The GHT is a reliable technique that, together with the Canny edge detector, fulfills the requirements of the problem. Furthermore, it offers the possibility of measuring the similarity between models and, this way, build around a classification system.

An analysis of the special features of this kind of images was performed. It shows that the complexity of the shapes and the high number of edges of these images are issues that deeply affect the performance. Different ways of designing the R-Table are suggested in order to avoid useless calculations, without success though. Nevertheless they could be employed for less complex shapes. The way of reducing the influence of the amount of edges is then addressed by the proposed preprocessing step. The reduction in edge points obviously depends on each image, but the resulting rates are high enough to speed up the whole process.
However, the preprocessing step is open to further extensions in order to increase the boundary points that were detected. This would be very useful for further applications, in order to better differentiate among all models in the database. Different incrementation strategies may be designed in this direction too. Higher values might be assigned to the increment of the voting process for beforehand defined interest points.

\section{References}

Ballard, D. H.: Generalizing the Hough Transform to Detect Arbitrary Shapes, Pattern Recognition, 13, 111-122, 1981.

Canny, J.: A Computational Approach to Edge Detection, IEEE T. Pattern Anal., 8, 697-714, 1986.

Duprat, O., Keck, B., Ruwwe, C., and Zölzer, U.: Hough Transform with weighting Edge-maps, Fifth IASTED International Conference on Visualization, Imaging, Image Processing (VIIP), Benidorm, Spain, 7-9 September 2005, 2005.

Hough, P. V. C.: Methods and Means for Recognising Complex Patterns, US Patent 3069 654, 1962.

Kassim, A. A., Tan, T., and Tan, K. H.: A Comparative Study of Efficient Generalised Hough Transform Techniques, Image Vision Comput., 17, 737-748, 1999.

Lee, H. M., Kittler, J., and Wong, K. C.: Generalised Hough Transform in Object Recognition, in: Proceedings of the 6th International Conference on Pattern Recognition, 285-289, 1992.

Tang, I. and Breckon, T. P.: Automatic Road Environment Classification, IEEE T. Intell. Transp., 12, 476-484, 2011. 